\title{
Pirfenidone attenuates expression of HSP47 in murine bleomycin-induced pulmonary fibrosis
}

\author{
T. Kakugawa*, H. Mukae*, T. Hayashi”, H. Ishii*, K. Abe*, T. Fujï, H. Oku, M. Miyazaki*, J. Kadota ${ }^{+}$, \\ S. Kohno*
}

Pirfenidone attenuates expression of HSP47 in murine bleomycin-induced pulmonary fibrosis. T. Kakugawa, H. Mukae, T. Hayashi, H. Ishii, K. Abe, T. Fujii, H. Oku, M. Miyazaki, J. Kadota, S. Kohno. (C) ERS Journals Ltd 2004.

ABSTRACT: Heat shock protein (HSP) 47, a collagen-specific molecular chaperone, is involved in the processing and/or secretion of procollagen. The present study was undertaken to investigate whether treatment with the antifibrotic drug pirfenidone attenuates the bleomycin (BL)-induced overexpression of HSP47 in the lungs.

Male ICR mice were intravenously injected with BL or saline (SA). Pirfenidone or control drug (CD) was administered 14 days after commencement of BL or SA, and continued throughout the course of the experiment. The mice were randomly divided into three experimental groups: 1) SA-treated with CD (SA group); 2) BL-treated with CD (BL group); and 3) BL-treated with pirfenidone (pirfenidone group).

Lungs of the pirfenidone group showed a marked reduction of fibrotic lesions compared with the corresponding BL group. Immunohistochemical studies showed that BL treatment significantly increased the number of macrophages, myofibroblasts, HSP47-positive type II pneumocytes and HSP47-positive interstitial spindle-shaped cells. Treatment with pirfenidone significantly reduced the number of these cells compared with the corresponding BL group. Furthermore, treatment with pirfenidone significantly suppressed the BL-induced increase of the positive ratio of HSP47 and $\alpha$ smooth muscle actin to interstitial spindle-shaped cells.

The present study results showed that pirfenidone inhibited heat shock protein 47positive cells and myofibroblasts, the principal cells responsible for the accumulation and deposition of extracellular matrix seen in pulmonary fibrosis. Eur Respir J 2004; 24: 57-65.
*Second Dept of Internal Medicine, Nagasaki University School of Medicine, " Dept of Pathology, Nagasaki University Hospital, Nagasaki, and "Medicinal Biology 3 Division, Discovery Research Laboratories, Shionogi \& Co. Ltd, Osaka, and ${ }^{+}$Second Dept of Internal Medicine, Oita Medical University, Oita, Japan.

Correspondence: H. Mukae, Second Dept of Internal Medicine, Nagasaki University School of Medicine, 1-7-1 Sakamoto, Nagasaki 8528501, Japan.

Fax: 81958497285

E-mail: hmukae@net.nagasaki-u.ac.jp

Keywords: Heat shock protein 47, myofibroblast, pirfenidone, pulmonary fibrosis, $\alpha$ smooth muscle actin

Received: October 282003

Accepted after revision: March 92004

This study was supported in part by a research grant from the Ministry of Education, Science, Sports and Culture of Japan.
Idiopathic pulmonary fibrosis (IPF) is a devastating disorder characterised by excess deposition of extracellular matrix (ECM) [1]. Increased synthesis and deposition of ECM is considered to be an important cause of fibrosis in experimental and human lung diseases [2]. However, the underlying molecular mechanism(s) responsible for the excessive deposition of collagen in the fibrotic lesions is not fully understood.

Heat shock protein (HSP) 47, first described as a collagenspecific stress protein, is thought to participate in intracellular processing, folding, assembly and secretion of procollagens [3-6]. Irrespective of the tissue site and organ, induction of HSP47 expression is always noted during the process of fibrosis, particularly in and around fibrotic lesions in both humans and experimental models [7, 8]. In pulmonary fibrosis, the HSP47-positive cells are thought to be the main source of collagen synthesis [4-9]. In addition, animal models of pulmonary fibrosis have confirmed the emergence of myofibroblasts in areas of active fibrosis, which diminish significantly as fibrosis wanes [10, 11]. Thus, myofibroblasts are also thought to play a central role in the synthesis, deposition and remodelling of the ECM in pulmonary fibrosis [9-12].

IPF remains a devastating clinical disorder for which there are limited therapeutic options. Although a number of experimental approaches have been investigated in animal models, including the inhibition of key cytokines and growth factors [13], the currently available therapeutic measures for treatment of IPF are clearly ineffective [14], and associated with significant adverse events and morbidity [15]. Pirfenidone, an antifibrotic agent, is known to inhibit progression of fibrosis in animal models and IPF patients [16-18]. Previous clinical studies with pirfenidone in IPF demonstrated its effectiveness in extending survival time and stabilising pulmonary function [18]. However, the mechanisms by which this new compound offers protection against lung fibrosis are not fully understood.

In the present study, the hypothesis that pirfenidone could attenuate the bleomycin (BL)-induced increase of HSP47positive cells and myofibroblasts followed by subsequent reduction of collagen accumulation was tested using a mouse model of BL-induced pulmonary fibrosis.

\section{Methods}

\section{Treatment of animals}

Male 10-week-old Institute for Cancer Research mice (Charles River Japan, Inc., Yokohama, Japan) weighing 36-39 g were specifically pathogen-free and maintained under standard conditions with free access to drinking water and pelleted food at the Animal Centre of Biomedical Research, 
Nagasaki University School of Medicine, Nagasaki, Japan. The mice were intravenously injected with $10 \mathrm{mg} \cdot \mathrm{kg}^{-1} \cdot \mathrm{day}^{-1}$ of BL (Nippon Kayaku, Tokyo, Japan) dissolved $\left(2 \mathrm{mg} \cdot \mathrm{mL}^{-1}\right)$ in isotonic saline solution (SA) or SA $\left(5 \mathrm{~mL} \cdot \mathrm{kg}^{-1} \cdot \mathrm{day}^{-1}\right)$ for 5 consecutive days. The animals were then treated orally twice daily with either pirfenidone (Shionogi \& Co., Osaka, Japan) at a dose of $400 \mathrm{mg} \cdot \mathrm{kg}^{-1} \cdot \mathrm{day}^{-1}$ dissolved in $0.5 \%$ carboxymethylcellulose (CMC) $\left(40 \mathrm{mg} \cdot \mathrm{mL}^{-1}\right)$, or with CMC $\left(10 \mathrm{~mL} \cdot \mathrm{kg}^{-1} \cdot \mathrm{day}^{-1}\right)$ alone as a control, starting 14 days after the commencement of i.v. injections of $\mathrm{BL}$ or $\mathrm{SA}$ and continuing throughout the course of the experiment. The mice were randomly divided into three experimental groups: SA-treated with control drug (CD) (SA group); BL-treated with CD (BL group); and BL-treated with pirfenidone (pirfenidone group). At 14 days after the commencement of BL or SA treatment, just before starting the administration of pirfenidone or $\mathrm{CD}$, mice were sacrificed and evaluated in the manner described below. At 35 and 49 days after the commencement of BL or SA treatment, mice were sacrificed and evaluated in the same way.

The experimental protocol was approved by the Ethics Review Committees for Animal Experimentation of Nagasaki University School of Medicine.

\section{Antibodies}

Primary antibodies used for the immunohistochemical studies included anti- $\alpha$-smooth muscle actin (SMA) (Neomarkers, Fremont, CA, USA), anti-F4/80 (Serotec, Oxford, UK), antisurfactant protein (SP)-A (Santa Cruz Biotechnology, Santa Cruz, CA, USA) and anti-HSP47 (Biotechnologies Corp., Victoria, BC, Canada). $\alpha$-SMA, F4/80 and SP-A were used as markers for myofibroblasts, macrophages and type II pneumocytes, respectively. Negative control studies were performed by using irrelevant immunoglobulin $\mathrm{G}$ with the same subclass of the first antibodies instead of the primary antibodies, and revealed no positive cells (data not shown).

\section{Histology and immunohistochemistry}

At 14, 35 and 49 days after the commencement of BL or SA injection, mice from each group $(n=3$ in each group at each time point) were sacrificed by exsanguination under deep anaesthesia (sodium pentobarbital, $50 \mathrm{mg} \cdot \mathrm{kg}^{-1}$, i.p.). Lungs were then removed from each mouse via a midline incision and were fixed in 10\% formaldehyde/neutral buffer solution, embedded in paraffin and processed to obtain $4-\mu \mathrm{m}$ sections for staining with haematoxylin-eosin. Sequential lung sections were placed on glass slides for immunohistochemical analysis.

The severity of fibrosis was evaluated semiquantitatively using a predetermined scale of severity (Ashcroft score [19]) $(n=3$ in each group at each time point). The entire lung section was examined at a magnification of $\times 100$. For each of $>15$ microscopic fields required to review the section, a numerical fibrosis score ranging from 0 (normal) to 8 (most severe) was assigned. The degree of cellular infiltration and fibrosis in the microscopic lung sections was assessed as the mean score for the observed fields in each sample.

Immunohistochemistry was performed with the conventional avidin-biotin-peroxidase histochemical technique using Histomouse $^{\text {TM }}$-Plus Kits (Zymed Laboratories, South San Francisco, CA, USA) for HSP47 and $\alpha$-SMA, and the Vecstain Elite ABC Kit (Vector Laboratories, Burlingame, CA, USA) for F4/80 and SP-A. Briefly, toluene was used to remove the paraffin from sequestered paraffin sections $(4 \mu \mathrm{m}$ thick) and the sections were rinsed thoroughly with ethanol. Sections were then soaked in $0.3 \% \mathrm{H}_{2} \mathrm{O}_{2}$ with absolute methanol for $20 \mathrm{~min}$ at room temperature to inactivate the endogenous peroxidase activity. They were incubated with blocking serum for $30 \mathrm{~min}$, and then covered with primary antibodies and incubated for $1 \mathrm{~h}$. After washing in phosphate-buffered saline, sections were processed further using kits according to the instructions provided by the manufacturer, and then developed with 3,3'-diaminobenzidine and $\mathrm{H}_{2} \mathrm{O}_{2}$, followed by the Mayer's haematoxylin staining method.

The number of HSP47-, $\alpha$-SMA- and F4/80-positive cells was counted at $\times 400$ magnification on lung specimens obtained from each group $(n=3$ in each group at each time point). Every five fields along the subpleural area of the lung, specimens were examined, resulting in a total of $\geqslant 10$ fields from each slide being counted. The number of interstitial spindle-shaped cells was also counted in the same manner, and the proportions of HSP47- and $\alpha$-SMA-positive interstitial spindle-shaped cells to all interstitial spindle-shaped cells were calculated ( $n=3$ in each group at each time point). Positive cell counts and proportions were expressed as the average number of cellular profiles per field, and the average proportions of HSP47- and $\alpha$-SMA-positive interstitial spindle-shaped cells to all interstitial spindle-shaped cells per field ( $\times 400$ magnification) of lung tissue section per mouse \pm SD. Histological sections were assessed independently, twice by each of two observers who were blind to the experimental groups. Interobserver and intraobserver variabilities were analysed using nested analysis of variance, and no significant difference was found among the results for all indices. Therefore, the results were considered to be reproducible with negligible interobserver and intraobserver variabilities. Statistical analysis was applied to representative results of one observer. Mean values of lungs of the BL group were compared with the corresponding values of the SA and pirfenidone groups.

\section{Measurement of lung hydroxyproline content}

To estimate the total amount of collagen deposited in the lung as an indicator of pulmonary fibrosis, the hydroxyproline content of the right lung was measured in each group of mice at 14, 35 and 49 days after the commencement of BL or $\mathrm{SA}$ administration $(\mathrm{n}=3$ in each group at 14 and 35 days and $\mathrm{n}=5$ in each group at 49 days). After measurement of wet lung weight, a $10-\mathrm{mg}$ aliquot of dried lung homogenate was hydrolysed in $6 \mathrm{~N} \mathrm{HCl}$ at $110^{\circ} \mathrm{C}$ for $24 \mathrm{~h}$. The hydrolysed specimen was fixed with nitrogen at $60^{\circ} \mathrm{C}$ for $20 \mathrm{~min}$ after filtration through a $0.45-\mu \mathrm{m}$ nylon membrane, dissolved in $12.5 \mathrm{mM}$ sodium borate, and prepared using fluorescamine solution. After centrifugation, the amount of hydroxyproline in the supernatant was measured using a capillary electrophoresis system (P/ACE MDQ; Beckman Coulter Inc., CA, USA).

\section{Statistical analysis}

All data values are expressed as mean \pm sD. Statistical differences among the SA group, BL group and pirfenidone group were analysed using one-way analysis of variance. The post hoc test used for multiple comparisons was the Fisher's paired least significant difference (PLSD) test. A value of $\mathrm{p}<0.05$ was considered statistically significant. 


\section{Results}

\section{Histological findings}

Histological examination revealed no significant changes in the lungs of the SA group at 14 (fig. 1a), 35 (data not shown) and 49 days (data not shown), while marked interstitial fibrosis was noted in the lungs of the BL group at 14 (fig. 1b), 35 (fig. 1c) and 49 days (fig. 1e). Lungs of the pirfenidone group exhibited a marked reduction in fibrotic areas at 35 (fig. 1d) and 49 (fig. 1f) days, relative to those of the BL groups at corresponding time intervals. At 14 days, just
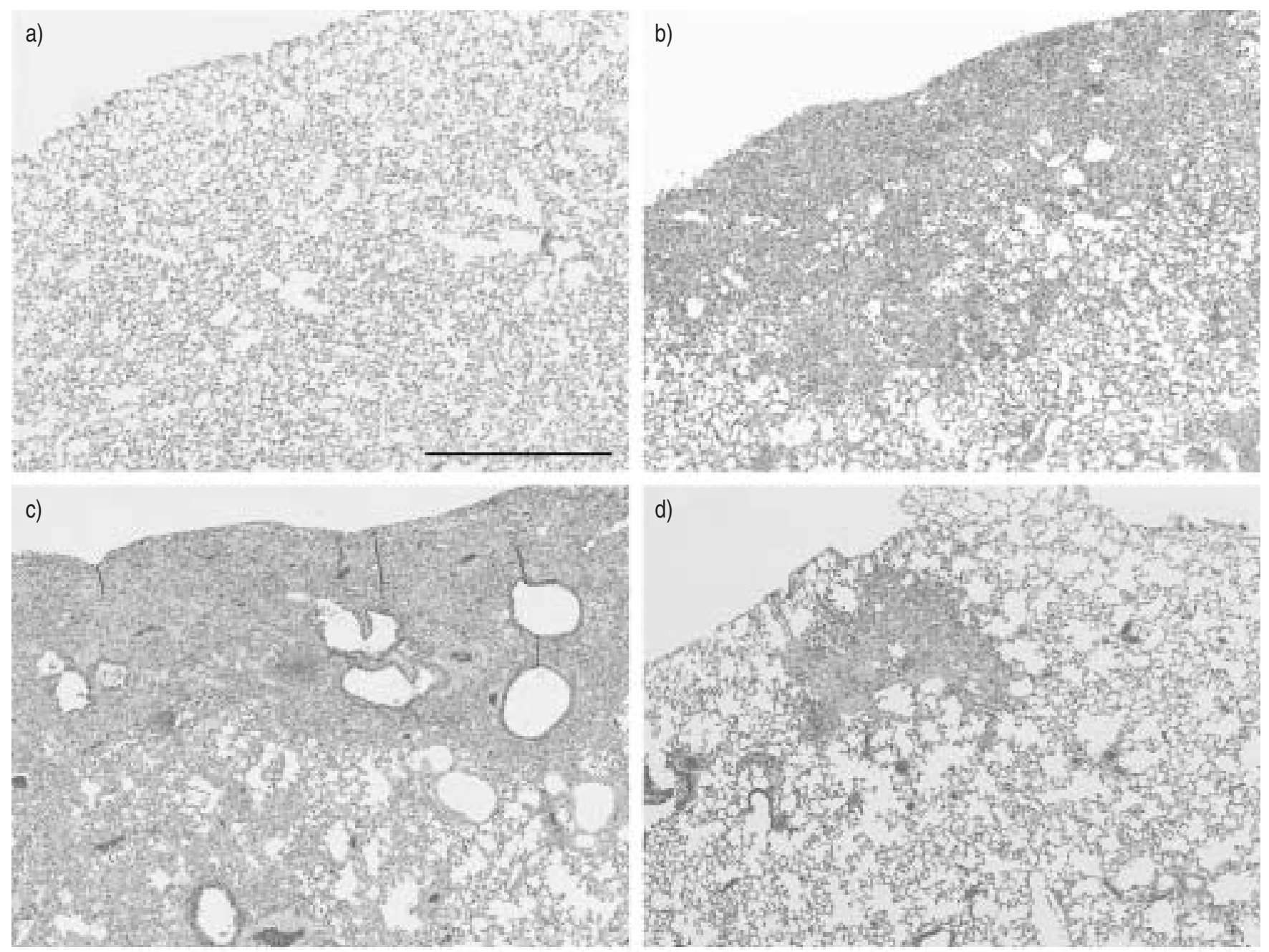

e)

f)
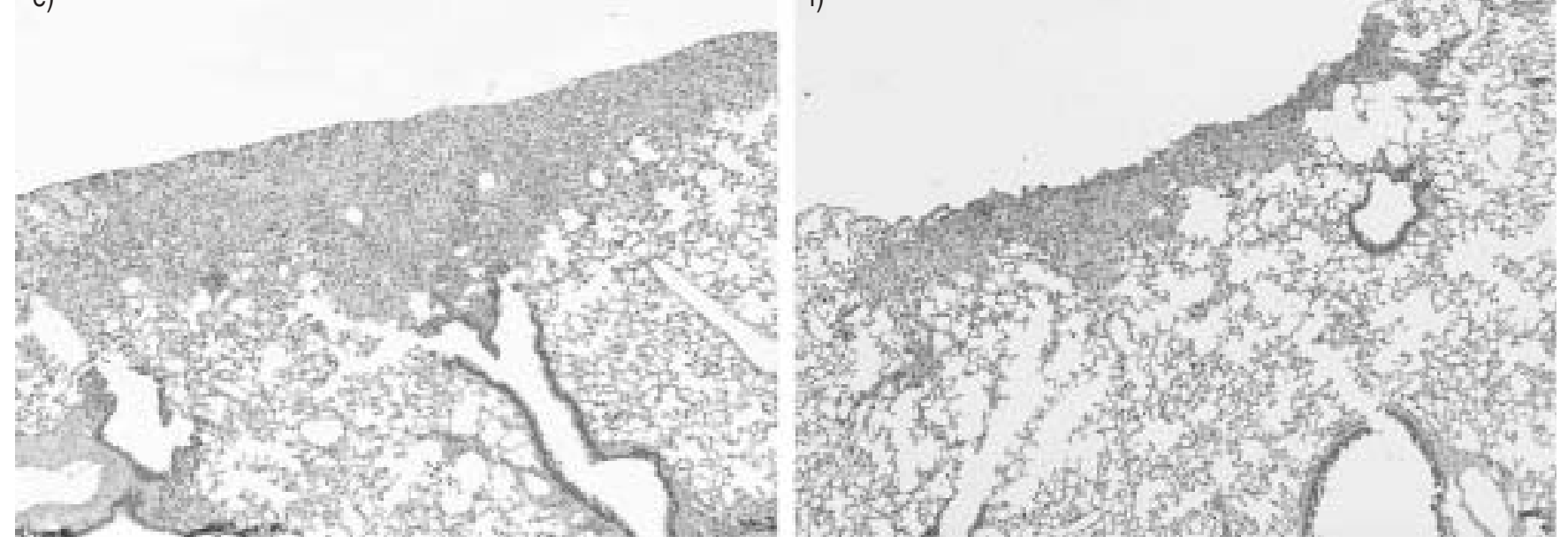

Fig. 1.- Representative photographs of the lungs for each group at different time intervals (haematoxylin and eosin stain). At 14 days, just before starting to administer pirfenidone or control drug, histological examination revealed no significant changes in the lungs of the saline group (a), while a marked interstitial fibrosis was noted in the lungs of the bleomycin (BL) group (b). Marked interstitial fibrosis was noted in the lungs of the BL group at 35 (c) and 49 days (e). Lungs of the pirfenidone group showed a marked reduction of fibrotic lesions at 35 (d) and 49 days (f). Scale bar=500 $\mu \mathrm{m}$. 


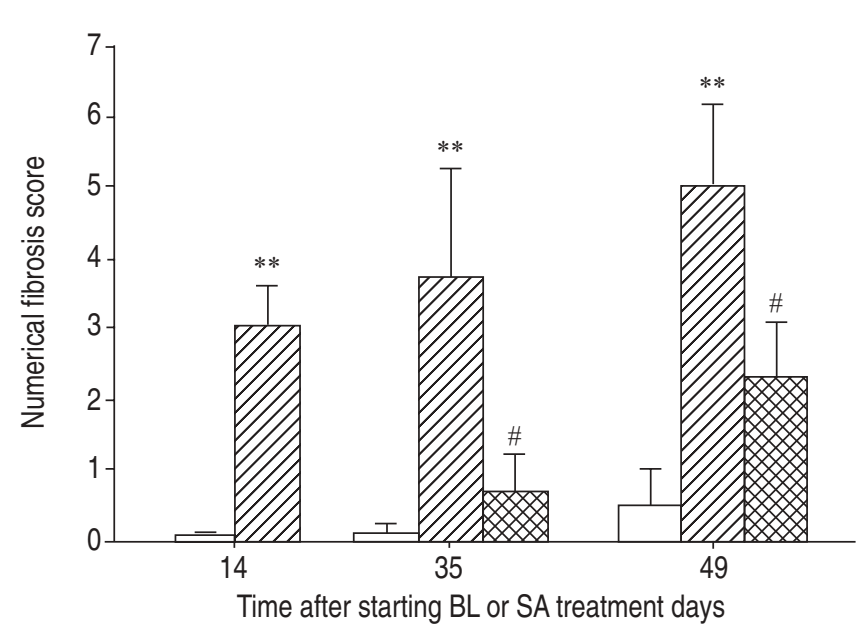

Fig. 2. - Effects of pirfenidone on bleomycin (BL)-induced pulmonary fibrosis at different time intervals. Data are presented as mean \pm SD.

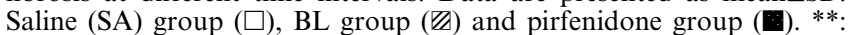
$\mathrm{p}<0.01$ compared with other groups at corresponding time intervals; \#: $\mathrm{p}<0.01$ compared with the $\mathrm{BL}$ group at corresponding time intervals.

before starting to administer pirfenidone or $\mathrm{CD}$, the mean fibrosis score in the BL group $(3.07 \pm 0.54)$ was significantly higher $(p<0.01)$ than that in the SA group $(0.04 \pm 0.04)$. The mean fibrosis scores in the BL group at 35 and 49 days $(3.74 \pm 1.53$ and $5.02 \pm 1.15$, respectively) were each significantly higher $(p<0.01)$ than in the corresponding values of the SA group $(0.09 \pm 0.09$ and $0.48 \pm 0.55$ at 35 and 49 days, respectively). Furthermore, treatment with pirfenidone caused a significant reduction $(\mathrm{p}<0.01$, each) in the mean fibrosis score of the pirfenidone group $(0.66 \pm 0.58$ and $2.35 \pm 0.77$ at 35 and 49 days, respectively) compared with the corresponding values of the BL group (fig. 2).

\section{Lung hydroxyproline content}

Figure 3 demonstrates the effect of pirfenidone on the hydroxyproline content, an index of collagen accumulation, at various time intervals. BL treatment $(356.85 \pm 83.47$,

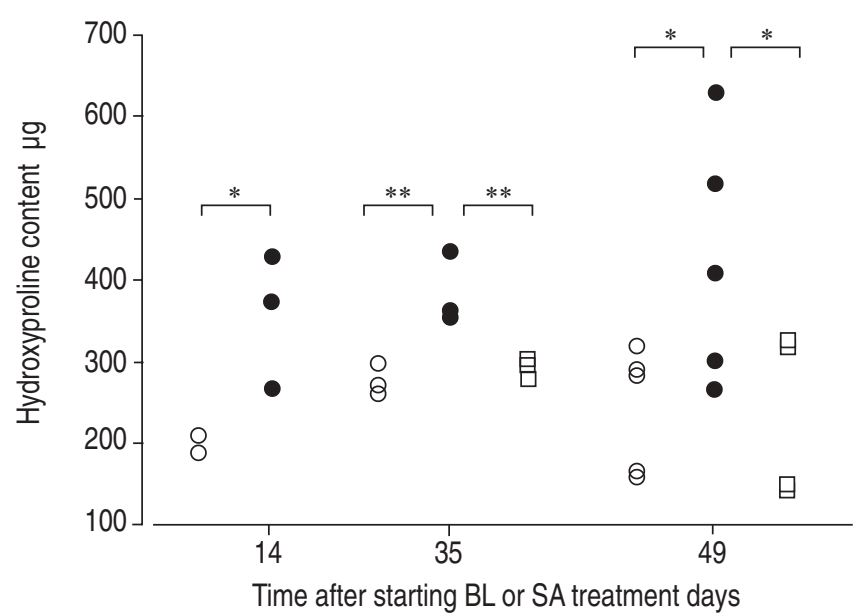

Fig. 3. - Effects of pirfenidone on bleomycin (BL)-induced increase of hydroxyproline content, an index of collagen accumulation at different time intervals. Saline (SA) group $(\bigcirc)$, BL group $(\bullet)$ and pirfenidone group ( $\square$ ). *: $p<0.05 ; * *: p<0.01$.
$384.60 \pm 43.63$ and $424.72 \pm 149.91 \mu \mathrm{g}$ at 14,35 and 49 days, respectively) significantly increased ( $\mathrm{p}<0.01$ at 35 days, and $\mathrm{p}<0.05$ at 14 and 49 days) the hydroxyproline content compared with the SA group (198.62 $\pm 12.77,275.71 \pm 17.02$ and $245.26 \pm 75.31 \mu \mathrm{g}$ at 14,35 and 49 days, respectively). Treatment with pirfenidone caused a significant reduction ( $\mathrm{p}<0.01$ at 35 days and $\mathrm{p}<0.05$ at 49 days) in the lung hydroxyproline content $(288.33 \pm 9.29$ and $252.56 \pm 94.60 \mu \mathrm{g}$ at 35 and 49 days, respectively), relative to the corresponding values of the BL-treated animals. Indeed, there was no significant difference in the hydroxyproline content between the SA group and pirfenidone group at corresponding time intervals.

\section{Immunohistochemistry}

At 35 and 49 days, only a few cells positive for F4/80, $\alpha$ SMA were detected in the lungs of the SA group (fig. 4a for F4/80 and fig. $4 \mathrm{~b}$ for $\alpha$-SMA at 49 days). In contrast, the number of F4/80-positive macrophages and $\alpha$-SMA-positive myofibroblasts increased markedly in the active fibrotic area in the BL group (fig. $4 \mathrm{c}$ for F4/80 and fig. $4 \mathrm{~d}$ for $\alpha$-SMA at 49 days). Treatment with pirfenidone resulted in a significant reduction in the number of these positive cells at the corresponding time intervals (fig. $4 \mathrm{e}$ for F4/80 and fig. 4f for $\alpha$-SMA at 49 days).

Only a few cells positive for HSP47 were detected in the lungs of the SA-treated group at 14 (fig. 5a), 35 (data not shown) and 49 (fig. 5c) days. In contrast, the number of HSP47-positive cells increased markedly in the active fibrotic area in the BL group at 14 (fig. 5b), 35 (data not shown) and 49 (fig. 5d) days. To determine the type of cells that expressed HSP47, the present study performed immunohistochemistry for $\alpha$-SMA and SP-A, in addition to staining for HSP47 using mirror image sections in the BL group at 14 days. The majority of HSP47-positive interstitial spindle-shaped cells (fig. 5e, arrows) were also positive for $\alpha$-SMA (fig. $5 \mathrm{f}$, the mirror image section of that in Fig. 5e, arrows) indicating that these cells were myofibroblasts. Some of the HSP47positive cuboidal cells located in alveolar walls (fig. $5 \mathrm{~g}$, arrows) were also positive for SP-A (fig. 5h, the mirror image section of that in fig. $5 \mathrm{~g}$, arrows), indicating that these cells were type II pneumocytes. HSP47-positive interstitial spindle-shaped cells (fig. 5i) and type II pneumocytes (fig. 5j, arrows) were markedly increased in the active fibrotic areas in the BL group at 49 days. Treatment with pirfenidone led to a significant reduction in these positive cells at 35 (data not shown) and 49 days (fig. 5k and fig. 5l; arrows indicate type II pneumocytes).

\section{Effect of pirfenidone on F4/80-positive macrophages}

BL treatment significantly increased $(\mathrm{p}<0.01)$ the mean number of $\mathrm{F} 4 / 80$ positive macrophages $(59.96 \pm 2.65,77.33 \pm$ 20.96 and $81.68 \pm 10.88$ at 14,35 and 49 days, respectively) per field $(\times 400$ magnification $)$ in the BL group compared to their corresponding SA group $(27.10 \pm 6.55,27.94 \pm 13.39$ and $15.27 \pm 7.93$ at 14,35 and 49 days, respectively). At 35 days, there was no significant difference in the number of macrophages between the BL group and pirfenidone group (51.69 \pm 9.92$)$. However, at 49 days, treatment with pirfenidone significantly suppressed $(\mathrm{p}<0.01)$ the number of macrophages $(26.49 \pm 14.88)$ in pirfenidone group compared with the BL group (fig. 6). 

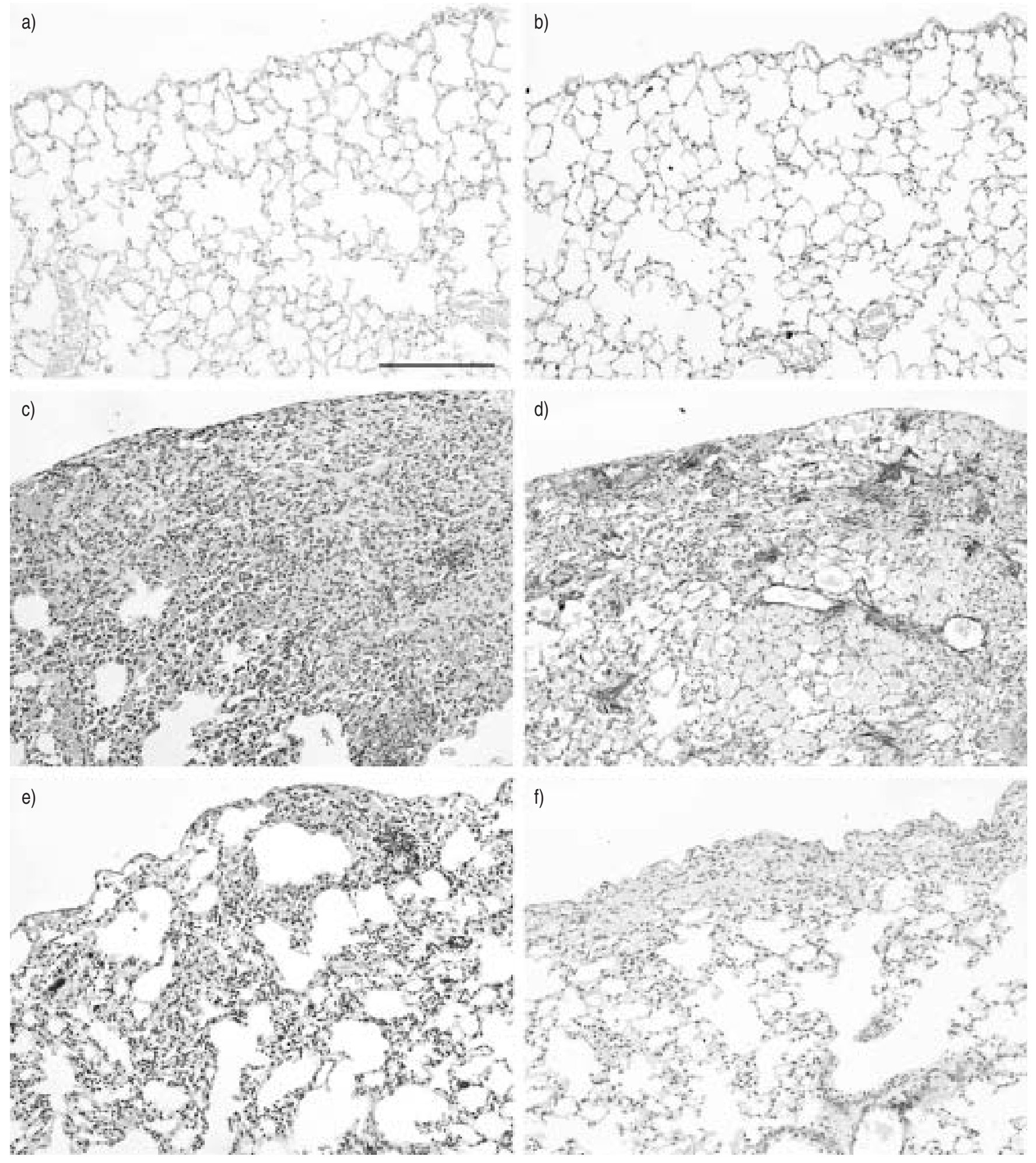

Fig. 4. - Immunohistochemical evaluation of saline (SA), bleomycin (BL) and pirfenidone groups at 49 days. Only a few F4/80-positive macrophages (a) and $\alpha$-smooth muscle actin (SMA)-positive myofibroblasts (b) were observed in the lungs of the SA group. In contrast, F4/80positive macrophages (c) and $\alpha$-SMA-positive myofibroblasts (d) were markedly increased in active fibrotic areas in the BL group. Treatment with pirfenidone caused a significant reduction in the BL-induced increase of F4/80 positive-macrophages (e) and $\alpha$-SMA-positive myofibroblasts (f) in the pirfenidone group. Scale bar $=200 \mu \mathrm{m}$.

\section{Effect of pirfenidone on the number of HSP47-positive interstitial spindle-shaped cells}

BL treatment significantly increased $(\mathrm{p}<0.01$ at 14 and 35 days, and $\mathrm{p}<0.05$ at 49 days) the mean number of
HSP47-positive interstitial spindle-shaped cells (7.50 \pm 2.00 , $35.67 \pm 12.80$ and $30.09 \pm 17.99$ at 14,35 and 49 days, respectively) per field ( $\times 400$ magnification) compared with the corresponding values of the SA group $(0.05 \pm 0.08,0.05 \pm 0.08$ and $0.05 \pm 0.08$ at 14,35 and 49 days, respectively). In 

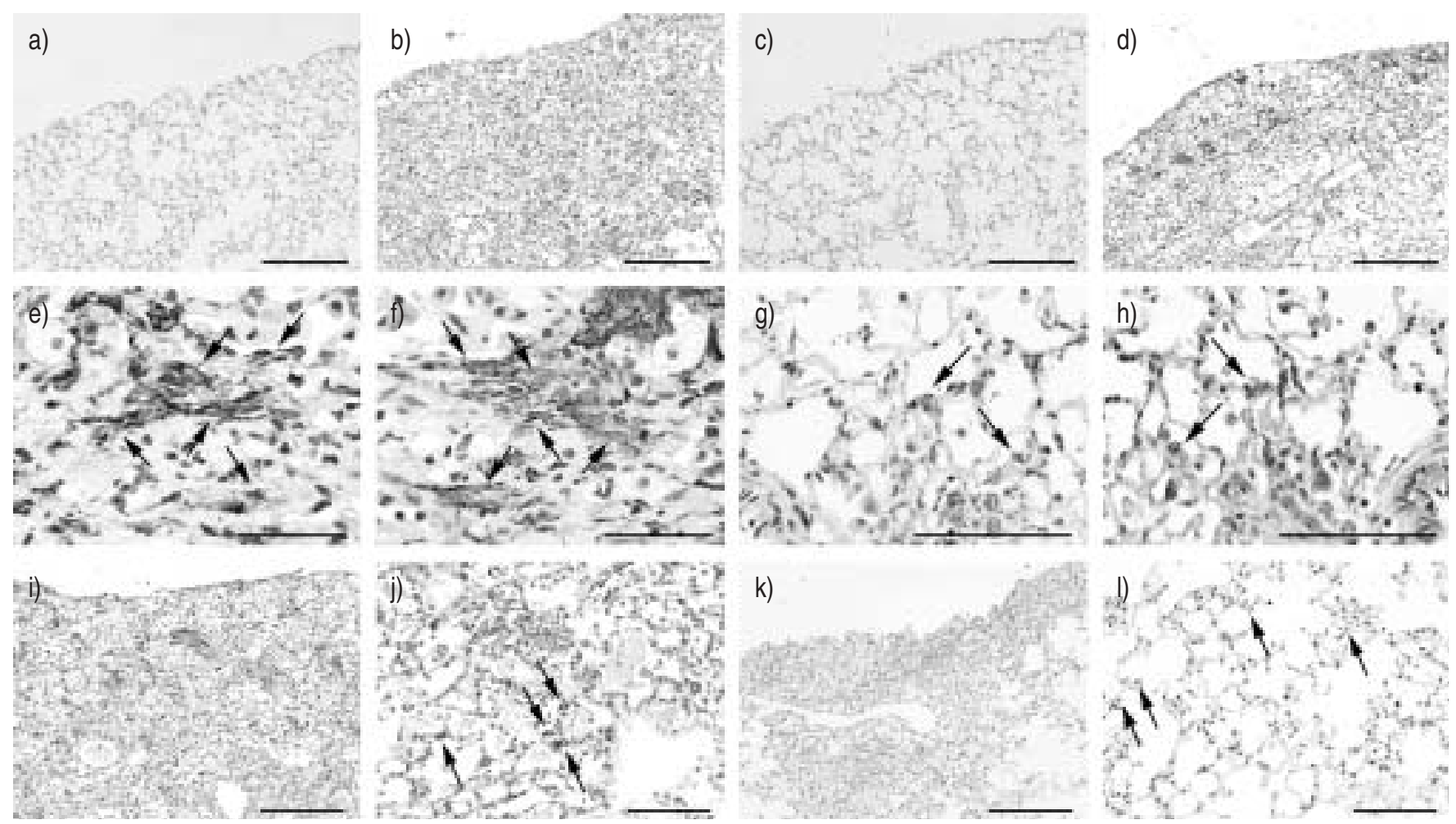

Fig. 5. - Immunohistochemical evaluation of saline (SA), bleomycin (BL) and pirfenidone groups. Only a few cells positive for heat shock protein (HSP) 47 were observed in the lungs of the SA group at 14 days (a) and 49 days (c). In contrast, HSP47-positive cells were markedly increased in active fibrotic areas in the BL group at 14 days (b) and 49 days (d). Mirror image sections to confirm the type of cells expressing HSP47 in the BL group at 14 days (e-h). The majority of the HSP47-positive interstitial spindle-shaped cells (e, arrows) were also positive for $\alpha$-smooth muscle action ( $\mathrm{f}$, the mirror image section of that in e, arrows), indicating that these cells are myofibroblasts. Some of the HSP47-positive cuboidal cells located in alveolar walls (g, arrows) were also positive for surfactant protein A ( $\mathrm{h}$, the mirror image section of that in $\mathrm{g}$, arrows), indicating that these cells are type II pneumocytes. HSP47-positive interstitial spindle-shaped cells (i) and type II pneumocytes (j, arrows) were markedly increased in active fibrotic areas in the BL group at 49 days. Treatment with pirfenidone caused a significant reduction in the BLinduced increase of HSP47-positive interstitial spindle-shaped cells (k) and type II pneumocytes (1, arrows). Scale bars $=200 \mu \mathrm{m}(\mathrm{a}-\mathrm{d}$, i, and k); $100 \mu \mathrm{m}(\mathrm{g}, \mathrm{h}, \mathrm{j}$ and $\mathrm{l})$; and $25 \mu \mathrm{m}$ (e and $\mathrm{f})$.

contrast, treatment with pirfenidone significantly suppressed ( $p<0.01$ at 35 days and $p<0.05$ at 49 days) the number of HSP47-positive interstitial spindle-shaped cells $(0.95 \pm 1.43$

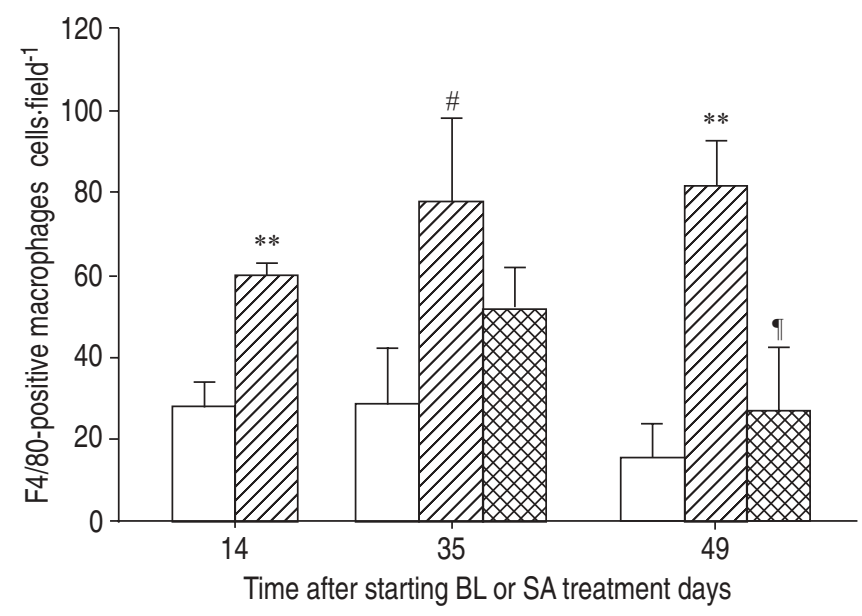

Fig. 6. - Effect of pirfenidone on bleomycin (BL)-induced increase of F4/80-positive macrophages. Data are presented as mean \pm SD. Saline

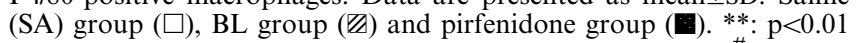
compared with the values at corresponding time interval; ${ }^{\#}: \mathrm{p}<0.01$ compared with the SA group at the corresponding time intervals; $"$ $\mathrm{p}<0.01$ compared with the $\mathrm{BL}$ group at the corresponding time intervals. and $3.87 \pm 1.40$ at 35 and 49 days, respectively) compared with the corresponding values of the $\mathrm{BL}$ group (fig. $7 \mathrm{a}$ ). BL treatment significantly increased $(\mathrm{p}<0.05$ at 14 days, and $\mathrm{p}<0.01$ at 35 and 49 days) the mean proportion of HSP47positive interstitial spindle-shaped cells to all interstitial spindle-shaped cells $(43.0 \pm 15.9,77.2 \pm 1.5$ and $72.8 \pm 12.5 \%$ at 14,35 and 49 days, respectively) relative to the corresponding values of the SA group (1.7 $\pm 2.9,2.2 \pm 3.9$ and $1.7 \pm 2.9 \%$ at 14,35 and 49 days, respectively). Treatment with pirfenidone significantly suppressed $(\mathrm{p}<0.01)$ the mean proportions of HSP47-positive interstitial spindle-shaped cells (11.5 \pm 16.7 and $34.3 \pm 10.4 \%$ at 35 and 49 days, respectively) compared with the corresponding values of the BL group (fig. 7b).

\section{Effect of pirfenidone on the number of $\alpha$-SMA-positive myofibroblasts}

BL treatment significantly increased $(\mathrm{p}<0.05$ at 14 and 49 days, and $\mathrm{p}<0.01$ at 35 days) the mean number of $\alpha$-SMApositive myofibroblasts $(20.81 \pm 8.71, \quad 38.57 \pm 19.86$ and $25.20 \pm 15.13$ at 14,35 and 49 days, respectively) per field $(\times 400$ magnification) relative to the corresponding values of the SA group $(0.11 \pm 0.20,0.19 \pm 0.32$ and $0.48 \pm 0.83$ at 14,35 and 49 days, respectively). In contrast, treatment with pirfenidone significantly suppressed $(p<0.05)$ the mean number of $\alpha$-SMA-positive myofibroblasts (7.05 \pm 6.92 and $1.32 \pm 1.89$ at 35 and 49 days, respectively) compared with the 

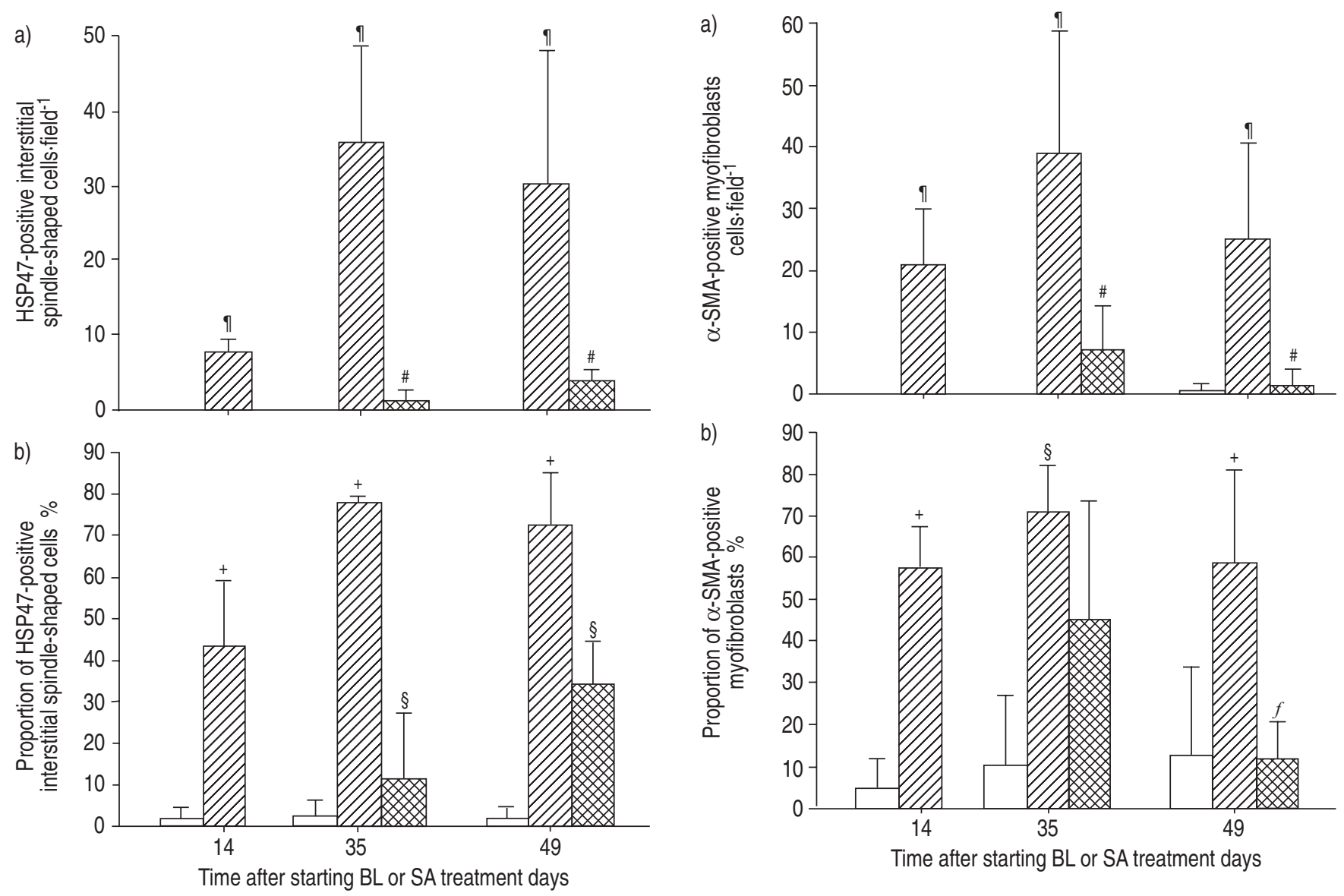

Fig. 7. - a) Effects of pirfenidone on the bleomycin (BL)-induced increase of the heat shock protein (HSP) 47-positive interstitial spindle-shaped cells. b) Effects of pirfenidone on the BL-induced increase of proportion of HSP47-positive interstitial spindle-shaped cells to all interstitial spindle-shaped cells (\%). Data are presented as mean \pm SD. Saline (SA) group $(\square)$, BL group $(\mathbb{Z})$ and pirfenidone group (田). $\because$ p $<0.01$ at 14 and 35 days and $\mathrm{p}<0.05$ at 49 days compared with the corresponding values of the other groups; \#: $\mathrm{p}<0.01$ at 35 days and $\mathrm{p}<0.05$ at 49 days compared with the BL group; ${ }^{+}: \mathrm{p}<0.05$ at 14 days and $\mathrm{p}<0.01$ at 35 and 49 days compared with the corresponding values of the other groups; \$: $p<0.01$ compared with the BL group at corresponding time intervals.

corresponding values of the BL group (fig. 8a). BL treatment significantly increased $(\mathrm{p}<0.01$ at 14 and 35 days and $\mathrm{p}<0.05$ at 49 days) the mean proportion of $\alpha$-SMA-positive interstitial spindle-shaped cells, myofibroblasts, to all interstitial spindle-shaped cells $(57.5 \pm 9.4,71.3 \pm 10.2$ and $58.6 \pm 22.6 \%$ at 14,35 and 49 days, respectively) relative to the corresponding values of the SA group $(4.2 \pm 7.3,9.8 \pm 17.0$ and $12.3 \pm 21.4 \%$ at 14,35 and 49 days, respectively). At 35 days, there was no significant difference in the mean proportion of $\alpha$-SMA-positive myofibroblasts between the BL and pirfenidone groups $(45.0 \pm 28.5 \%)$. At 49 days, treatment with pirfenidone significantly suppressed $(\mathrm{p}<0.05)$ the mean proportion of $\alpha$-SMA-positive myofibroblasts $(11.4 \pm 9.1 \%)$ compared with the BL group (fig. $8 \mathrm{~b}$ ).

\section{Discussion}

Pirfenidone, an antifibrotic agent, is known to inhibit progression of fibrosis in animal models $[16,17]$. Pirfenidone is an investigational drug currently being evaluated for its

Fig. 8. -a) Effect of pirfenidone on bleomycin (BL)-induced increase of $\alpha$-smooth muscle actin (SMA)-positive myofibroblasts. b) Effects of pirfenidone on BL-induced increase of the proportion of $\alpha$-SMApositive myofibroblasts to all interstitial spindle-shaped cells (\%). Data are presented as mean \pm SD. Saline (SA) group ( $\square$ ), BL group $(\mathbb{Z})$ and pirfenidone group $(\because)$. $p<0.05$ at 14 and 49 days and $\mathrm{p}<0.01$ at 35 days compared with the corresponding values of the other groups; \#: $\mathrm{p}<0.05$ compared with the BL group at corresponding time intervals; ${ }^{+}: \mathrm{p}<0.01$ at 14 days and $\mathrm{p}<0.05$ at 49 days compared with the corresponding values of the other groups; \$: $\mathrm{p}<0.01$ compared with the corresponding values of the SA group; ${ }^{f}$ : $\mathrm{p}<0.05$ compared with the BL group at corresponding time intervals.

ability to affect lung fibrosis in human subjects. The agent was previously reported to be effective in extending survival time and stabilising pulmonary functions in IPF patients in a pilot clinical study, with an open-label design [18]. However, the precise mechanisms by which this new compound offered protection against lung fibrosis are not fully understood.

HSP47 is a collagen-binding, stress-inducible protein localised in the endoplasmic reticulum, and is never released into the ECM. HSP47 has a specific role only in the intracellular processing of procollagen production as a collagenspecific molecular chaperone [4-6]. The present authors have previously reported that HSP47 plays a key role in increased deposition of collagens in usual interstitial pneumonia (UIP) [9], fibrotic transplanted kidney [7] and peritoneal sclerosis [8]. Recent reports have demonstrated that HSP47 expression is highly tissue- and cell-specific, restricted to mostly phenotypically altered collagen-producing cells, and its expression correlates well with that of collagen [7-9]. It was demonstrated that inhibition of HSP47 by antisense oligodeoxynucleotides markedly suppressed the production of collagen in 3T6 cells [6], in experimental proliferative glomerulonephritis [20] and in experimental peritoneal fibrosis [21]. These findings suggest the important role of 
HSP47 in collagen synthesis in various fibrotic disorders. Although extensive work is still needed at this stage, HSP47 seems to be a promising target for the treatment of fibrotic diseases.

Activation of $\alpha$-SMA-positive myofibroblasts are also believed to be a central event that plays a key role in the progression of chronic pulmonary fibrosis. ZHANG et al. [11] showed that $\alpha$-SMA expression coincides with procollagen gene expression in BL-induced pulmonary fibrosis in rats. Recently, the current authors reported that patients with UIP provide evidence that myofibroblasts in fibroblast foci can express procollagen type I protein and HSP47 [9]. These findings suggest that increased myofibroblasts contribute to the fibrosis process through the production of HSP47-induced type I procollagen [9-12]. The current study also showed that BL treatment induced a marked increase of HSP47 and myofibroblasts in active fibrotic areas of the lung from the early stage of fibrotic process at 14 days. In addition, pirfenidone inhibited HSP47-positive cells and myofibroblasts that had already increased and proliferated during the process of fibrosis, which might result in the attenuation of pulmonary fibrosis.

A logical therapeutic approach to pulmonary fibrosis would be down-regulation of HSP47-positive cells and myofibroblasts through induction of apoptosis, as occurs in normal wound healing $[22,23]$. Another approach is changing the phenotype of HSP47-positive cells and myofibroblasts. In this regard, it is interesting that treatment with pirfenidone decreased the ratios of HSP47 and $\alpha$-SMA to all interstitial spindle-shaped cells, suggesting that pirfenidone might change the phenotype of fibroblasts.

Increased contractility of the affected tissue with consequent impairment of respiratory mechanics and function is thought to be another role of myofibroblasts [24, 25]. Recently, a pilot clinical study, with an open-label design using pirfenidone for the treatment of IPF, demonstrated the effectiveness of this compound in stabilising pulmonary function [18]. The present findings imply that a marked reduction in the number of contractile myofibroblasts in the lungs might contribute to stabilising the deterioration of pulmonary function in those patients.

Previous studies indicated that marked disruption of the integrity of the alveolar epithelium and presence of several altered phenotypes are distinctive features of various stages of IPF. Disarrangement of the lung epithelium in IPF is an important defect that hinders appropriate re-epithelisation [13]. SELman et al. [13] reported that IPF involves abnormal wound healing in response to multiple microscopic sites of ongoing alveolar epithelial injury and activation associated with the formation of patchy fibroblast-myofibroblast foci, which evolve to fibrosis. The present authors previously reported that regenerated type II pneumocytes in UIP start expressing type I procollagen through the induction of HSP47 and play an important role in the development of fibrosis [9]. Recently, the present authors have also shown that type II pneumocytes start expressing HSP47 as fibrosis proceeds, in a rodent BL-induced pulmonary fibrosis model [26]. These findings suggest that increased type II pneumocytes, in addition to myofibroblasts, contribute to the fibrotic process through the production of HSP47-induced type I procollagen and suggest that HSP47-positive type II pneumocytes might represent a suitable target for therapeutic strategies designed for chronic pulmonary fibrosis. It is noteworthy that treatment with pirfenidone also decreased HSP47-positive type II pneumocytes that had already increased from the early stage of fibrosis. Although the number of HSP47-positive type II pneumocytes was not counted due to the general difficulty in counting cells in the remodelled fibrotic area of the lung, differences among the groups were evident.
The intrapulmonary sequestration of macrophages in the lungs of BL-treated mice was suppressed by pirfenidone treatment. This is an interesting finding since macrophages are known to play a critical role in the pathogenesis of pulmonary fibrosis by producing a variety of cytokines such as interleukin (IL)-1, tumour necrosis factor- $\alpha$, transforming growth factor (TGF)- $\beta$ and platelet derived growth factor (PDGF) [27, 28]. Demouliere et al. [29] demonstrated that TGF- $\beta$ mediates the attainment of myofibroblastic features, including $\alpha$-SMA expression, by cultured skin fibroblasts. TANG et al. [30] showed that infusion of PDGF induced tubulointerstitial myofibroblast formation in rats. The present authors previously reported that infiltrating macrophages might be involved in the induction of HSP47 expression in fibroblasts in renal fibrosis [7]. The results of these early studies, together with the present findings, imply that other possible antifibrotic mechanisms for pirfenidone might include the ability to decrease intrapulmonary sequestration of macrophages.

The observation that pirfenidone attenuates overexpression of HSP47 and $\alpha$-SMA in animals after the initiation of pulmonary injury, may have significant implications for the development of clinically relevant therapeutic strategies for pulmonary fibrosis. It is possible that administration of pirfenidone may be therapeutically effective for chronic pulmonary fibrosis through inhibition of HSP47-positive cells and $\alpha$-SMA-positive myofibroblasts, the principal cells responsible for the accumulation and deposition of ECM seen in pulmonary fibrosis.

A complete interpretation of the present pathological study is limited because the current study did not elucidate whether changes in heat shock protein-47 and $\alpha$-smooth muscle actin are the direct effects of pirfenidone or are secondary to an overall inhibition of fibrosis. Further in vitro studies using cell lines are necessary to elucidate the precise mechanism of action of pirfenidone on the expression of heat shock protein47 and $\alpha$-smooth muscle actin.

\footnotetext{
Acknowledgements. The authors thank M. Mine, associate professor in the Biostatistics Section, Division of Scientific Data Registry, Atomic Bomb Disease Institute, Nagasaki University Graduate School of Biomedical Sciences, Nagasaki, Japan, for excellent statistical analysis. The authors also thank A. Yokoyama for excellent technical support.
}

\section{References}

1. Crouch E. Pathobiology of pulmonary fibrosis. Am J Physiol 1990; 259: L159-L184.

2. Specks U, Nerlich A, Colby TV, Wiest I, Timpl R. Increased expression of type VI collagen in lung fibrosis. Am J Respir Crit Care Med 1995; 151: 1956-1964.

3. Nakai A, Satoh M, Hirayoshi K, Nagata K. Involvement of the stress protein HSP47 in procollagen processing in the endoplasmic reticulum. J Cell Biol 1992; 117: 903-914.

4. Nagata K, Saga S, Yamada KM. A major collagen-binding protein of chick embryo fibroblasts is a novel heat shock protein. J Cell Biol 1986; 103: 223-229.

5. Saga S, Nagata K, Chen WT, Yamada KM. pH-dependent function, purification, and intracellular location of a major collagen-binding glycoprotein. J Cell Biol 1987; 105: 517527.

6. Sauk JJ, Smith T, Norris K, Ferreira L. Hsp47 and the translation-translocation machinery cooperate in the production of alpha 1(I) chains of type I procollagen. $J$ Biol Chem 1994; 269: 3941-3946. 
7. Abe K, Ozono Y, Miyazaki M, et al. Interstitial expression of heat shock protein 47 and alpha-smooth muscle actin in renal allograft failure. Nephrol Dial Transplant 2000; 15: 529-535.

8. Shioshita K, Miyazaki M, Ozono Y, et al. Expression of heat shock proteins 47 and 70 in the peritoneum of patients on continuous ambulatory peritoneal dialysis. Kidney Int 2000; 57: 619-631.

9. Iwashita $\mathrm{T}$, Kadota $\mathrm{J}$, Naito $\mathrm{S}$, et al. Involvement of collagen-binding heat shock protein 47 and procollagen type I synthesis in idiopathic pulmonary fibrosis: contribution of type II pneumocytes to fibrosis. Hum Pathol 2000; 31: $1498-1505$.

10. Vyalov SL, Gabbiani G, Kapanci Y. Rat alveolar myofibroblasts acquire alpha-smooth muscle actin expression during bleomycin-induced pulmonary fibrosis. Am J Pathol 1993; 143: 1754-1765.

11. Zhang K, Rekhter MD, Gordon D, Phan SH. Myofibroblasts and their role in lung collagen gene expression during pulmonary fibrosis. A combined immunohistochemical and in situ hybridization study. Am J Pathol 1994; 145: 114-125.

12. Kuhn C, McDonald JA. The roles of the myofibroblast in idiopathic pulmonary fibrosis. Ultrastructural and immunohistochemical features of sites of active extracellular matrix synthesis. Am J Pathol 1991; 138: 1257-1265.

13. Selman M, King TE, Pardo A. Idiopathic pulmonary fibrosis: prevailing and evolving hypotheses about its pathogenesis and implications for therapy. Ann Intern Med 2001; 134: 136-151.

14. Hunninghake GW, Kalica AR. Approaches to the treatment of pulmonary fibrosis. Am J Respir Crit Care Med 1995; 151: 915-918.

15. Lynch JP, 3rd, McCune WJ. Immunosuppressive and cytotoxic pharmacotherapy for pulmonary disorders. $\mathrm{Am}$ J Respir Crit Care Med 1997; 155: 395-420.

16. Iyer SN, Margolin SB, Hyde DM, Giri SN. Lung fibrosis is ameliorated by pirfenidone fed in diet after the second dose in a three-dose bleomycin-hamster model. Exp Lung Res 1998; 24: 119-132.

17. Iyer SN, Gurujeyalakshmi G, Giri SN. Effects of pirfenidone on procollagen gene expression at the transcriptional level in bleomycin hamster model of lung fibrosis. $J$ Pharmacol Exp Ther 1999; 289: 211-218.

18. Raghu G, Johnson WC, Lockhart D, Mageto Y. Treatment of idiopathic pulmonary fibrosis with a new antifibrotic agent, pirfenidone: results of a prospective, open-label Phase II study. Am J Respir Crit Care Med 1999; 159: 1061-1069.

19. Ashcroft T, Simpson JM, Timbrell V. Simple method of estimating severity of pulmonary fibrosis on a numerical scale. J Clin Pathol 1988; 41: 467-470.

20. Sunamoto M, Kuze K, Tsuji H, et al. Antisense oligonucleotides against collagen-binding stress protein HSP47 suppress collagen accumulation in experimental glomerulonephritis. Lab Invest 1998; 78: 967-972.

21. Nishino T, Miyazaki M, Abe K, et al. Antisense oligonucleotides against collagen-binding stress protein HSP47 suppress peritoneal fibrosis in rats. Kidney Int 2003; 64: 887-896.

22. Desmouliere A, Redard M, Darby I, Gabbiani G. Apoptosis mediates the decrease in cellularity during the transition between granulation tissue and scar. Am J Pathol 1995; 146: 56-66.

23. Desmouliere A, Badid C, Bochaton-Piallat ML, Gabbiani G. Apoptosis during wound healing, fibrocontractive diseases and vascular wall injury. Int J Biochem Cell Biol 1997; 29: 19-30.

24. Majno G, Gabbiani G, Hirschel BJ, Ryan GB, Statkov PR Contraction of granulation tissue in vitro: similarity to smooth muscle. Science 1971; 173: 548-550.

25. Evans JN, Kelley J, Low RB, Adler KB. Increased contractility of isolated lung parenchyma in an animal model of pulmonary fibrosis induced by bleomycin. Am Rev Respir Dis 1982; 125: 89-94.

26. Ishii $\mathrm{H}$, Mukae $\mathrm{H}$, Kakugawa $\mathrm{T}$, et al. Increased expression of collagen-binding heat shock protein 47 in murine bleomycin-induced pneumopathy. Am J Physiol Lung Cell Mol Physiol 2003; 285: L957-963.

27. Khalil N, Bereznay O, Sporn M, Greenberg AH. Macrophage production of transforming growth factor beta and fibroblast collagen synthesis in chronic pulmonary inflammation. J Exp Med 1989; 170: 727-737.

28. Martinet Y, Rom WN, Grotendorst GR, Martin GR, Crystal RG. Exaggerated spontaneous release of plateletderived growth factor by alveolar macrophages from patients with idiopathic pulmonary fibrosis. N Engl J Med 1987; 317 : 202-209.

29. Desmouliere A, Geinoz A, Gabbiani F, Gabbiani G. Transforming growth factor-beta 1 induces alpha-smooth muscle actin expression in granulation tissue myofibroblasts and in quiescent and growing cultured fibroblasts. $J$ Cell Biol 1993; 122: 103-111.

30. Tang WW, Ulich TR, Lacey DL, et al. Platelet-derived growth factor-BB induces renal tubulointerstitial myofibroblast formation and tubulointerstitial fibrosis. Am J Pathol 1996; 148: 1169-1180. 